\title{
A Novel Molecular Model of Plant Lectin-Induced Programmed Cell Death in Cancer
}

\author{
Zheng Shi, ${ }^{a, \#}$ Wen-wen Li, ${ }^{b, \#}$ Yong Tang, ${ }^{*, c}$ and Li-jia Cheng*,a \\ ${ }^{a}$ School of Medicine, Chengdu University; Chengdu 610106, China: ${ }^{b}$ UCL Institute of Ophthalmology; \\ 11-43 Bath Street, London ECIV 9EL, U.K.: and ${ }^{c}$ School of Acupuncture and Tuina, Chengdu \\ University of Traditional Chinese Medicine; Chengdu 610075, China. \\ Received May 1, 2017; accepted July 20, 2017; advance publication released online August 1, 2017
}

\begin{abstract}
Plant lectin, a class of highly diverse non-immune origin and carbohydrate-binding proteins, has been reported to specially induce cancer cell through programmed cell death (PCD) pathways (apoptosis and/or autophagy), shedding lights on screening promising anti-cancer candidate agent for further therapeutic trials. However, the complicated molecular mechanisms by which plant lectins induced the programmed death of tumor cells, have not yet been fully clarified. Here, we summarized a novel model, based on vast amount of research, by which plant lectins eliminate various types of cancer cells via three major pathways, including a) direct ribosome inactivating, b) endocytosis-dependent mitochondrial dysfunction and c) sugar-containing receptors binding. A better understanding of the role of plant lectins played and further elucidation of the strategies targeting PCD would provide a new clue for the applications and modifications of plant lectin as a potential anti-cancer agent from bench to clinic.
\end{abstract}

Key words plant lectin; programmed cell death; molecular model; autophagy; apoptosis; drug development

\section{INTRODUCTION}

Plant lectins, also known as phytohemagglutinin, are complexes of oligosaccharides and proteins, which contains at least one non-catalytic domain for selective recognizing and agglutinating cells reversibly, or for precipitating polysaccharose and glycoconjugates through the free glycans on glycoproteins and glycolipids without altering the structure of carbohydrate. ${ }^{1)}$ Based on the molecular structures and evolutionary statuses, plant lectins are further categorized into twelve families, namely Amaranthin, Agaricus bisporus agglutinin, Cyanovirin, Chitinase-related agglutinin, Euonymus europaeus agglutinin, Galanthus nivalis agglutinin (GNA), Jacalins, Lysin motif, Hevein, Legume lectin, Nictaba, and Ricin-B families, respectively. ${ }^{2)}$ These 12 plant lectin families are well-known to possess extensive biological functions, such as anti-fungal, anti-viral and anti-neoplastic activities. ${ }^{3,4)}$

Over the last two decades, owing to the binding specificities, researchers have utilized plant lectins to recognize the subtle distinctions between malignant cells and non-malignant cells to discriminate tumor from benign and the degree of glycolsylation associated with metastasis. ${ }^{5)}$ Specially, lectin-glycan interactions have critical functions in a variety of physiological and pathological processes, including immune inflammatory responses and tumor progression. Lectin-glycan recognition systems have been reported to facilitate the initiation, execution and resolution of cell death, functioning domains of representative lectins and their carbohydrate specificity were presented in Table $\left.1 .{ }^{6}\right)$

A few plant lectins, such as mistletoe lectin (ML) have been adopted for alternative cancer therapy in clinical trials (Gastric cancer, phase 4; non-small-cell lung cancer, phase 2). ${ }^{13)}$ In addition, ML has been reported to reduce the side-effects as

\footnotetext{
${ }^{\#}$ These authors contributed equally to this work.
}

adjuvant agents during radiotherapy and chemotherapy. ${ }^{14)}$ With in-depth investigations, plant lectins, as sophisticated microarray reagents for specially recognizing tumor in diagnosis, treatment and prognosis of cancer, so far have generated more attention due to their remarkable anti-tumor activities toward a variety of cancer cells. ${ }^{15,16)}$

To date, plant lectins have been wildly reported to inhibit pro-survival pathways (e.g., angiogenesis, Wnt signaling, and protein synthesis), and especially induce apoptosis as well as autophagy, by which cancer undergo programmed cell death (PCD) ${ }^{17-19)}$ However, the specific mechanisms of which remain nubilous. After summarizing vast amount of experimental and theoretical research, we proposed a model of how the plant lectins induce PCD.

\section{A NOVEL MODEL OF PLANT LECTIN-INDUCED PCD}

We observed that plant lectins could initiate cell death via three major pathways, including 1) direct ribosome inactivating, 2) endocytosis-dependent mitochondrial dysfunction 3) and/or sugar-containing receptors binding (Fig. 1).

2.1. Direct Ribosome Inactivation? MLs were classified as type II ribosome-activating proteins, which have drawn more and more attention owing to their unique anti-cancer abilities and therapeutically applications. Members in ML subfamily are normally consists of two chains: an A-chain containing three conserved individual domains, and a B-chain which transports A chain into the cytoplasm after binding to certain sugar-chains or sugar-containing receptors on the cellular outer membrane. ${ }^{20)}$ Subsequently, its A-chain inhibits protein synthesis byh inactivating $28 \mathrm{~S}$ ribosomes on endoplasmic reticulum (ER $)^{21)}$ (Fig. 1A).

2.2. Endocytosis-Dependent Mitochondrial Dysfunction? In addition, plant lectins sush as Concanavalin A

* To whom correspondence should be addressed. e-mail: tangyong@cdutcm.edu.cn; chenglijia@cdu.edu.cn 
Table 1. Overview of Several Typical Plant Lectins Carbohydrate-Binding Motifs

\begin{tabular}{lll}
\hline \hline \multicolumn{1}{c}{ Lectin domain } & \multicolumn{1}{c}{ Carbohydrate specificity } & \multicolumn{1}{c}{ Examples } \\
\hline Galanthus nivalis agglutinin domain & $\begin{array}{l}\text { Man, oligomannosides, high-man } N \text {-glycans, complex, } \\
N \text {-glycans }\end{array}$ & GNA, ASA II, ASAL, ACA, LOA \\
Agaricus bisporus agglutinin domain & T-Antigen & ABA, MarpoABA \\
Nicotiana tabacum agglutinin domain & GlcNAc-oligomers, high-man $N$-glycans & NICTABA, PP2 \\
Legume lectin domain & Man/Glc, Gal/GalNAc, $($ GlcNAc)n, Fuc, Siaa2 $\rightarrow 3$ Gal/ & PHA, ConA, Gleheda, PSA, GSII \\
& GalNAc, Complex $N$-glycans & 7 \\
Hevein domain & Chitin, high-man, Man, $N$-glycans & Hevein, UDA, WGA, HFR3 \\
Ricin-B domain & Gal/GalNAc, Siaa2 $\rightarrow 6$ Gal/GalNAc & Ricin, SNA-I \\
Jacalins & Gal, T-antigen, Man, $N$-glycans & Jacalin, Heltuba, HFR1 \\
Amaranthins & T-Antigen & Amaranthin, HFR2 \\
Class V chitinase homologs & Blood group B, high-man $N$-glycans & RobpsCRP \\
Euonymus europaeus agglutinin & Blood group B, high-man $N$-glycans & EEA \\
domain & & 10 \\
\hline
\end{tabular}

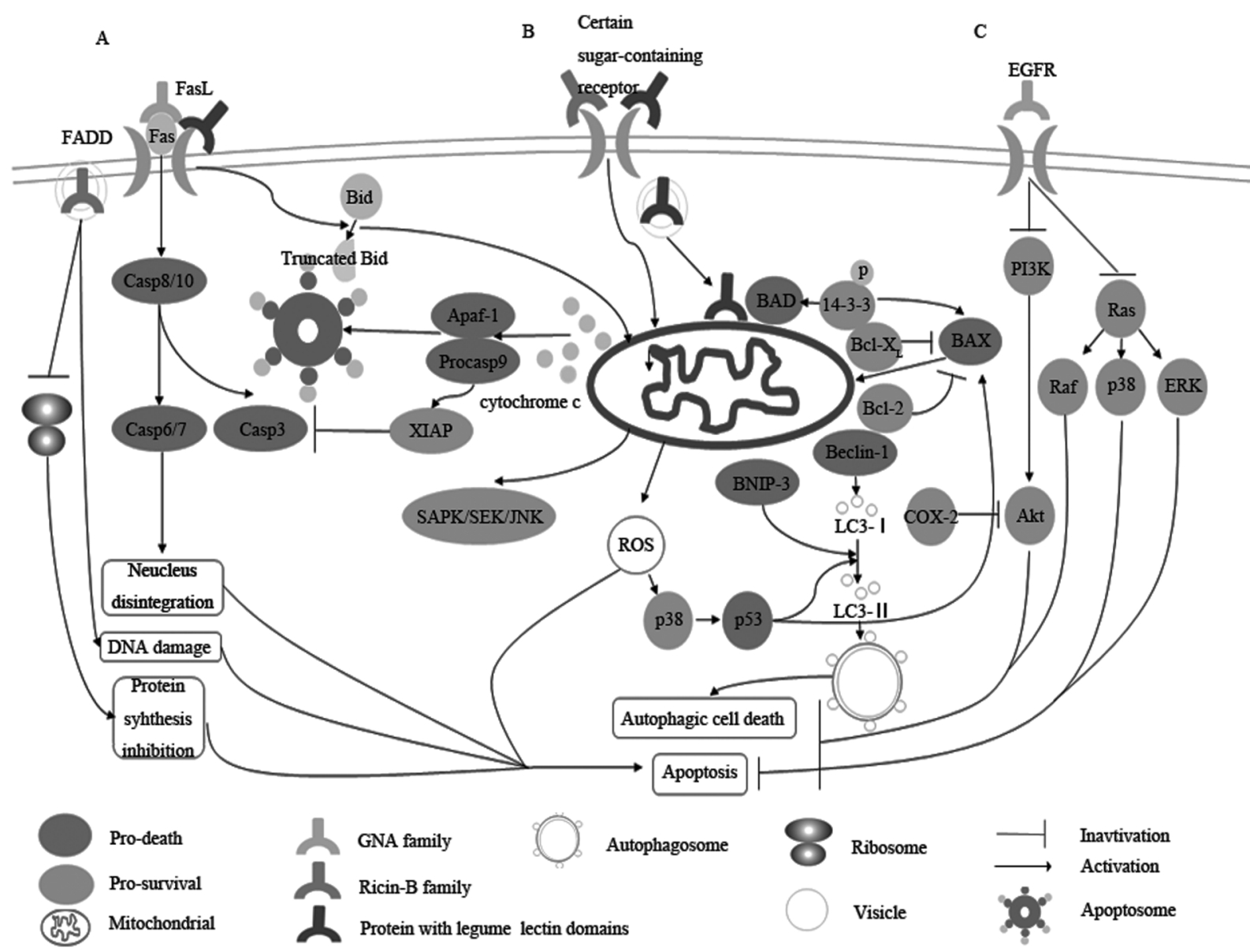

Fig. 1. A Summarized Molecular Model for Plant Lectin-Induced PCD

(A) MLs and Ricin-B family lectin beard apoptosis-inducing activities towards cancer cells by targeting different stages of apoptotic pathways via directly inactivate ribosome. (B) Some lectins, taking ConA for example, depended on endocytosis and selectively localize on mitochondrion in cancer cell, and autophagic cell death was initiated. (C) Some lectins such as ConA and PCL bind certain sugar-containing receptors on the surface of cancer cells, subsequently apoptosis was triggered.

(ConA), could be internalized to and subsequently bind to the mitochondria via clathrin-mediated endocytosis after binding to mannose moiety of the glycoproteins on the cellular membrane (Fig. 1B).

The internalized plant lectins were reported to result in a significant decrease of mitochondrial membrane potential (MMP), and thus releasing the cytochrome $c$ (Cyto $c$ ) which eventually sparks off apoptosis. The reduction of mitochondrial transmembrane potential was also reported to initiate autophagy via the inductions of Bcl2/adenovirus E1B $19 \mathrm{kDa}-$ interacting protein 3 (BNIP3) or Beclin-1.22,23)

2.3. Binding Sugar-Containing Receptors on the Cell Surface? Interestingly, the third way is less-known. Several plant lectins, such as ConA and Polygonatum cyrtonema lectin (PCL) are reported to bind to the sugar-containing receptors on the cell surface to directly inhibit anti-death/survival pathways (Fig. 1C), suggesting sugar-binding specificity might be one of the main reasons motivating the anti-tumor activity. $^{24,25)}$

In this model, three pathways are closely interlinked. The 
ML from Ricin-B family that own A- and B-chains, bind to the membrane receptors before inactivated the ribosome. ${ }^{26}$ ) ConA, the most representative legume lectin, could bind to matrix metalloproteinase family receptors on cell membrane to inhibit angiogenesis, establish immunological memory via antigens, as well as induce PCD through endocytosis. ${ }^{27)}$ Interestingly, the endocytosis-dependent PCD related to lectinreceptors binding on mitochondria outer membrane. As for the PCL of GNA family, PCL-Fas binding would initiate caspasedependent apoptosis, similarly the PCL-epidermal growth factor receptor (EGFR) binding inhibits both apoptotic and autophagic cell death. ${ }^{28)}$

\section{PREDICTIONS AND OBSERVATIONS OF THE NEW MODEL}

3.1. Activating Pro-apoptotic and Blocking Anti-apoptotic Pathway In contrast to necrosis, apoptosis is a highly regulated and controlled process of cell death which controls cell proliferation or functions as a defense systems in response to failure of DNA damage repairmen. As one of the 12 major anti-cancer mechanisms, plant lectins activate pro-apoptotic and blocks anti-apoptotic pathways in cancer cell models. ${ }^{29)}$

The ML-1 and other Ricin-B family members' stimulation initiated apoptosis via extrinsic pathway by activating caspase- 8 in Jurkat leukemic T cells, and mitochondria-mediated pathway independently of p53 in hepatocarcinoma cells. Khil et al. reported Korean mistletoe lectin broke down MMP in mitochondrial pathway, releasing Cyto $c$ as well as increasing of the level of reactive oxygen species (ROS), and thus activating caspase-3 to form apoptosome in COLO cells. ${ }^{30)}$ Feng et al. reported that ML-1 activated transforming growth factor (TGF)- $\alpha$, dephosphorylated Bcl-2 independently of p21 and p53 to promote apoptosis. Chinese mistletoe lectin-I was reported to down-regulate the expression of miR-135a, b and up-regulate the levels of target gene APC; thereby, decreasing the downstream Wnt signaling activity in CLY and HT-29 cells. ${ }^{31)}$ Moreover, ML-1-A-Chain activated jun N-terminal kinase (JNK) dissociated BAX and BAD from 14-3-3 by translocating the pro-apoptotic proteins, including Bax and Bad to mitochondria. ${ }^{32)}$ In addition to ML-1, ML-2 induced apoptosis via mitogen-activated protein kinases (MAPK) signaling, activation of SAPK/JNK and p38 pathways as well as inhibition of extracellular-signal regulated kinases $1 / 2$ (ERK1/2) pathway. Korean mistletoe lectin was reported to kill COLO cells in a time-dependent and dose-dependent manner by activating caspases and inhibiting expression of anti-apoptotic molecules (e.g., nuclear factor-kappaB (NF- $\kappa \mathrm{B}), \mathrm{X}$-linked inhibitor-ofapoptosis protein (XIAP), and Akt/protein kinase B). ${ }^{33)}$

For proteins with legume lectin domains, tumor cells underwent mitochondria-dependent apoptosis. ${ }^{34)}$ MMP collapses upon legume lectin treatments, procaspase-3 turned into active caspase- 3 which conjugated with apoptosome resulting in conditions of irreparable DNA damage. However, in p53-null cells, ConA induced apoptosis through activation of $\mathrm{p} 73$. The tumor suppressor p73 played a significant role in rendering the ration of Bax/Bcl-2, blocking the survival Akt pathway, as well as activating Foxola-Bim signaling that execute apoptosis. Furthermore, via activation of IKK-NF- $\kappa$ B-cyclooxygenase (COX)-2， SHP-2-MEK-1-ERK， SHP-2-Ras-ERK signaling, ConA was reported inhibit survival in tumor cell model. ${ }^{35,36)}$
Under the treatment of PCL, apoptosis was induced with the activation of caspase- $3,-8,-9 .{ }^{37)}$ The PCL treatment also abrogated the glutathione antioxidant system, and promoted ROS accumulation that subsequently activated p38 and p53signaling. ${ }^{38)}$ In addition, PCL further promoted apoptosis by blocking Ras-Raf and phosphoinositide 3-kinase (PI3K)Akt pathways in L929 cells.

3.2. Promoting Autophagic Cell Death Pathways, with/ without Apoptosis Autophagy is an evolutionarily conserved mechanism for degradation and renovation of superfluous macro-complexes and in eukaryotic cells. Plant lectin would also promote cancer autophagic cell death with/without apoptosis. $^{39)}$

Liu and colleagues reported that ConA inducedautophagy only in hepatoma cells through BNIP3-mediated mitochondrial pathway. BNIP3 is a cell death-inducing factor whose main function is to determine the on/off state of the mitochondrial permeability transition pore. ${ }^{40)}$ Therefore, BNIP3 decreases membrane potential on molecular lever, and thus initiating autophagy after ConA internalizing to mitochondria surface. $^{41)}$

In contrast, Liu et al. reported that as a member of GNA, PCL induced autophagic death via activation of mitochondrial ROS-p38-p53 pathway in A375 cells, as well as via blocking Ras-Raf and PI3K-Akt pathways in L929 cells. ${ }^{42,43)}$ The signaling pathways discussed above, revealed an complicated relationship between apoptotic and autophagic death upon PCL treatments.

\section{VIA THIS MODEL, IS PLANT LECTIN A PROMISING ANTI-CANCER DRUG TARGETING PCD?}

Plant lectin has been reported to remarkably inhibit growth of a variety of cancer cell models. Since 1989, plant lectins have been used as a class of reliably biochemical, cytological, and histochemical probes to distinguish non-malignant from malignant cells that are otherwise non-detectable with available monoclonal antibodies. ${ }^{44)}$ Since 1993, plant lectins have drawn great attention by their remarkable PCD inducing activities, propelling the research of plant lectin to a new level. ${ }^{45)}$ Vast amount of studies have been carried out with the aim to clarify the specific molecular mechanisms or clinical benefits of plant lectins. ${ }^{17,46)}$ In addition, as a T cell mitogen in vivo, plant lectins killed cells by stimulating immune system. ${ }^{47)}$ An immune memory would be generated after plant lectin-induced eradication, therefore resisting to the same genotypic tumor.

However, is plant lectin a promising anti-cancer drug targeting PCD? Collectively, we believe the experimental results have been rather positive and promising. Several typical lectins, such as MLs, ricin and Phaseolus vulgaris lectin, have been utilized as potential anti-tumor drugs or adjuvant therapeutic agents in clinical treatment for over two decades, ${ }^{48}$ ) the majority of plant lectins are still cytotoxic. When plant lectins selectively kill cancer cells, there would be concomitant toxic effects on normal cell and animal model. It is worth mentioning that legume lectins have been reported to trigger TNF- $\alpha$-mediated hepatitis in mice. ${ }^{49)}$ The side-effects present a difficult challenge for further applications of plant lectin in medicine, although a great quantity of experiments have been dedicated to the molecular modification. ${ }^{50)}$ 


\section{CONCLUSION}

Based on extensive research have been carried out, three major pathways are summarized with the aim to unveil the mystery of plant lectin-induced PCD in this study, therefore providing basic guidance for pre-clinical and clinical trials. We believe a better understanding of the role plant lectin played and further elucidation of the strategies targeting PCD would provide a new clue for the further investigation of plant lectin as a potential anticancer drug candidate from bench to clinic.

Acknowledgments We thank Prof. Jinku-Bao (Sichuan University) and Tian Yu (Chengdu University) for technological assistance. W. Li was a China Scholarship Council (CSC) funded Ph.D. student. This work was supported in part by the Grants Scientific and Technological Funds for Young Scientists of Sichuan (2017JQ0060), the science and technology support program of Science \& Technology Department of Sichuan (2016NZ0060), Sichuan Provincial Innovative Research Team Program (2014TD0018, 2015TD0010), Undergraduate Training Program for Innovation and Entrepreneurship of Sichuan (201711079010, 201711079069), Innovative Research Team in University of Sichuan (16TD0015), and National Natural Science Foundation of China (81373735).

Conflict of Interest The authors declare no conflict of interest.

\section{REFERENCES}

1) Damme EJMV, Lannoo N, Peumans WJ. Plant lectins. Adv. Bot. Res., 48, 107-209 (2008).

2) Goldstein IJ, Hughes RC, Monsigny M, Osawa T, Sharon N. What should be called a lectin? Nature, 285, 66 (1980).

3) Yau T, Dan X, Ng CC, Ng TB. Lectins with potential for anticancer therapy. Molecules, 20, 3791-3810 (2015).

4) Sharon N, Lis H. History of lectins: from hemagglutinins to biological recognition molecules. Glycobiology, 14, 53R-62R (2004).

5) Sharon N, Lis H. Lectins as cell recognition molecules. Science, 246, 227-234 (1989).

6) Giacometti J. Plant lectins in cancer prevention and treatment. $\mathrm{Me}$ dicina, 51, 211-229 (2015).

7) Lichtenstein RG, Rabinovich GA. Glycobiology of cell death: when glycans and lectins govern cell fate. Cell Death Differ., 20, 976-986 (2013).

8) Kletter D, Singh S, Bern M, Haab BB. Global comparisons of lectin-glycan interactions using a database of analyzed glycan array data. Molecular \& Cellular Proteomics Mcp, 12, 1026-1035 (2013).

9) van EM, Bruinsma L, Hartshorn KL, White MR, Rynkiewicz MJ Seaton BA, Hemrika W, Romijn RA, van Balkom BW, Haagsman HP. Introduction of $N$-linked glycans in the lectin domain of surfactant protein D: impact on interactions with influenza a viruses. $J$. Biol. Chem., 286, 20137-20151 (2011).

10) Lorenz V, Ditamo Y, Cejas RB, Carrizo ME, Bennett EP, Clausen $\mathrm{H}$, Nores GA, Irazoqui FJ. Extrinsic functions of lectin domains in $\mathrm{O}-\mathrm{N}$-acetylgalactosamine glycan biosynthesis. J. Biol. Chem., 291, 25339-25350 (2016)

11) Lira-Navarrete E, de LRM, Compañón I, Pallarés MC, Kong Y, Iglesias-Fernández J, Bernardes GJL, Peregrina JM, Rovira C, Bernadó P, Bruscolini P, Clausen H, Lostao A, Corzana F, HurtadoGuerrero R. Dynamic interplay between catalytic and lectin domains of GalNAc-transferases modulates protein $O$-glycosylation.
Nat. Commun., 6, 6937 (2015).

12) Ielasi FS, Alioschaperez M, Donohue D, Claes S, Sahli H, Schols D, Willaert RG. Lectin-glycan interaction network-based identification of host receptors of microbial pathogenic adhesins. MBio, 7, e00584-e16 (2016)

13) Marvibaigi M, Supriyanto E, Amini N, Abdul Majid FA, Jaganathan SK. Preclinical and clinical effects of mistletoe against breast cancer. Biomed Research International, 2014, 785479 (2014).

14) Mody R, Joshi SHA, Chaney W. Use of lectins as diagnostic and therapeutic tools for cancer. J. Pharmacol. Toxicol. Methods, 33, 1-10 (1995)

15) Liu B, Bian HJ, Bao JK. Plant lectins: potential antineoplastic drugs from bench to clinic. Cancer Lett., 287, 1-12 (2010).

16) Coulibaly FS, Youan BBC. Current status of lectin-based cancer diagnosis and therapy. AIMS Molecular Science, 4, 1-27 (2017).

17) Jiang QL, Zhang S, Tian M, Zhang SY, Xie T, Chen DY, Chen YJ, He J, Liu J, Ouyang L, Jiang X. Plant lectins, from ancient sugarbinding proteins to emerging anti-cancer drugs in apoptosis and autophagy. Cell Prolif., 48, 17-28 (2015).

18) Lichtenstein RG, Rabinovich GA. Glycobiology of cell death: when glycans and lectins govern cell fate. Cell Death Differ., 20, 976-986 (2013).

19) Ouyang L, Shi Z, Zhao S, Wang FT, Zhou TT, Liu B, Bao JK. Programmed cell death pathways in cancer: a review of apoptosis, autophagy and programmed necrosis. Cell Prolif., 45, 487-498 (2012)

20) Kienle GS, Berrino F, Büssing A, Portalupi E, Rosenzweig S, Kiene $\mathrm{H}$. Mistletoe in cancer-A systematic review on controlled clinical trials. Eur. J. Med. Res., 8, 109-119 (2003).

21) Han SY, Hong CE, Kim HG, Lyu SY. Anti-cancer effects of enteric-coated polymers containing mistletoe lectin in murine melanoma cells in vitro and in vivo. Mol. Cell. Biochem., 408, 73-87 (2015).

22) Chang CP, Yang MC, Liu HS, Lin YS, Lei HY. Concanavalin A induces autophagy in hepatoma cells and has a therapeutic effect in a murine in situ hepatoma model. Hepatology, 45, 286-296 (2007).

23) Shi Z, An N, Zhao S, Li X, Bao JK, Yue BS. In silico analysis of molecular mechanisms of legume lectin-induced apoptosis in cancer cells. Cell Prolif., 46, 86-96 (2013).

24) $\mathrm{Xu} \mathrm{Z,} \mathrm{Zhou} \mathrm{X}$, Lu H, Wu N, Zhao H, Zhang L, Zhang W, Liang YL, Wang L, Liu Y, Yang P, Zha X. Comparative glycoproteomics based on lectins affinity capture of $N$-linked glycoproteins from human Chang liver cells and MHCC97-H cells. Proteomics, 7, 2358-2370 (2007).

25) Gorelik E, Galili U, Raz A. On the role of cell surface carbohydrates and their binding proteins (lectins) in tumor metastasis. Cancer Metastasis Reviews, 20, 245-277 (2001).

26) Li WW, Yu JY, Xu HL, Bao JK. Concanavalin A: a potential antineoplastic agent targeting apoptosis, autophagy and anti-angiogenesis for cancer therapeutics. Biochem. Biophys. Res. Commun., 414, 282-286 (2011)

27) Faheina-Martins GV, Silveira ALD, Cavalcanti BC, Ramos MV, Moraes MO, Pessoa C, Araújo DAM. Antiproliferative effects of lectins from Canavalia ensiformis and Canavalia brasiliensis in human leukemia cell lines. Toxicolo. in Vitro, 26, 1161-1169 (2012).

28) Shi Z, Chen J, Li C, An N, Wang Z, Yang S, Huang K, Bao J. Antitumor effects of concanavalin A and Sophora flavescens lectin in vitro and in vivo. Acta Pharmacol. Sin., 35, 248-256 (2014).

29) Shi Z, Sun R, Yu T, Liu R, Cheng LJ, Bao JK, Zou L, Tang Y. Identification of novel pathways in plant lectin-induced cancer cell apoptosis. Int. J. Mol. Sci., 17, 228 (2016).

30) Lyu SY, Choi SH, Park WB. Korean mistletoe lectin-induced apoptosis in hepatocarcinoma cells is associated with inhibition of telomerase via mitochondrial controlled pathway independent of p53. Arch. Pharm. Res., 25, 93-101 (2002).

31) Hong M, Wang N, Tan HY, Tsao SW, Feng Y. MicroRNAs and Chinese medicinal herbs: new possibilities in cancer therapy. Cancers, 
(Basel), 7, 1643-1657 (2015).

32) Li LN, Zhang HD, Zhi R, Yuan SJ. Down-regulation of some miRNAs by degrading their precursors contributes to anti-cancer effect of mistletoe lectin-I. Br. J. Pharmacol., 162, 349-364 (2011).

33) Hoessli DC, Ahmad I. Mistletoe lectins: carbohydrate-specific apoptosis inducers and immunomodulators. Curr. Org. Chem., 12, 918-925 (2008).

34) Liu B, Li CY, Bian HJ, Min MW, Chen LF, Bao JK. Antiproliferative activity and apoptosis-inducing mechanism of Concanavalin A on human melanoma A375 cells. Arch. Biochem. Biophys., 482, 1-6 (2009).

35) Liu B, Min MW, Bao JK. Induction of apoptosis by Concanavalin A and its molecular mechanisms in cancer cells. Autophagy, 5, 432$433(2009)$

36) Dan X, Ng TB, Wong JH, Chan YS, Cheung RC, Chan WY. A hemagglutinin isolated from Northeast China black beans induced mitochondrial dysfunction and apoptosis in colorectal cancer cells.. Biochimica et Biophysica Acta, 1863, 2201-2211 (2016).

37) Liu B, Wu JM, Li J, Liu JJ, Li WW, Li CY, Xu HL, Bao JK. Polygonatum cyrtonema lectin induces murine fibrosarcoma L929 cell apoptosis and autophagy via blocking Ras-Raf and PI3K-Akt signaling pathways. Biochimie, 92, 1934-1938 (2010).

38) Liu B, Cheng Y, Zhang B, Bian HJ, Bao JK. Polygonatum cyrtonema lectin induces apoptosis and autophagy in human melanoma A375 cells through a mitochondria-mediated ROS-p38-p53 pathway. Cancer Lett., 275, 54-60 (2009).

39) Tyagi N, Tyagi M, Pachauri M, Ghosh PC. Potential therapeutic applications of plant toxin-ricin in cancer: challenges and advances. Tumour Biol., 36, 8239-8246 (2015)

40) Fu L-L, Zhao X, Xu H-L, Wen X, Wang SY, Liu B, Bao JK, Wei YQ. Identification of microRNA-regulated autophagic pathways in plant lectin-induced cancer cell death. Cell Prolif., 45, 477-485 (2012).

41) Liu B, Zhang B, Min MW, Bian HJ, Chen LF, Liu Q, Bao JK.
Induction of apoptosis by Polygonatum odoratum lectin and its molecular mechanisms in murine fibrosarcoma L929 cells. Biochim. Biophys. Acta, 1790, 840-844 (2009).

42) Wang SY, Yu QJ, Bao JK, Liu B. Polygonatum cyrtonema lectin, a potential antineoplastic drug targeting programmed cell death pathways. Biochem. Biophys. Res. Commun., 406, 497-500 (2011).

43) Liu B, Cheng Y, Bian HJ, Bao JK. Molecular mechanisms of Polygonatum cyrtonema lectin-induced apoptosis and autophagy in cancer cells. Autophagy, 5, 253-255 (2009).

44) De Mejía EG, Prisecaru VI. Lectins as bioactive plant proteins: a potential in cancer treatment. Crit. Rev. Food Sci. Nutr., 45, 425445 (2005).

45) Yu K, Mamoru I, Yoriyuki N. Plant lectins in therapeutic and diagnostic cancer research zuotian monira pervin 1. International Journal of Plant Biology \& Research, 3, 27-3 (2015)

46) Jamal S, Lavanya V, Adil A M. Lectins-the promising cancer therapeutics. Oncobiol. Targets, 1, 12 (2014).

47) Maletzki C, Linnebacher M, Savai R, Hobohm U. Mistletoe lectin has a shiga toxin-like structure and should be combined with other Toll-like receptor ligands in cancer therapy. Cancer Immunol. Immunother., 62, 1283-1292 (2013).

48) de MEG, Dia VP. The role of nutraceutical proteins and peptides in apoptosis, angiogenesis, and metastasis of cancer cells. Cancer Metastasis Rev., 29, 511-528 (2010).

49) Li J, Xia Y, Liu T, Wang J, Dai W, Wang F, Zheng Y, Chen K, Li S, Abudumijiti H, Zhou Z, Wang J, Lu W, Zhu R, Yang J, Zhang H, Yin Q, Wang C, Zhou Y, Lu J, Zhou Y, Guo C. Protective effects of astaxanthin on ConA-induced autoimmune hepatitis by the $\mathrm{JNK} / \mathrm{p}-\mathrm{JNK}$ pathway-mediated inhibition of autophagy and apoptosis. PLOS ONE, 10, e0120440 (2015).

50) Kang YJ, Bang BR, Otsuka M, Otsu K. Tissue-specific regulation of $\mathrm{p} 38 \alpha$-mediated inflammation in ConA-induced acute liver damage. J. Immunol., 194, 4759-4766 (2015). 